\title{
Prenatal Specification and Target Induction Underlie the Enrichment of Calcitonin Gene-Related Peptide in the Trigeminal Ganglion Neurons Projecting to the Cerebral Vasculature
}

\author{
Kevin Horgan, Timothy P. O'Connor, and Derek van der Kooy \\ Neurobiology Research Group, Department of Anatomy, University of Toronto, Toronto, Ontario, Canada M5S 1A8
}

The neurotransmitter calcitonin gene-related peptide (CGRP) is enriched in the adult rat trigeminal visceral projection to the cerebral arteries compared both to other neurotransmitters in this projection and to the percentage of CGRPpositive trigeminal cells projecting to cutaneous targets. In colchicine-treated ganglia approximately $30 \%$ of adult trigeminal ganglion cells projecting to the middle cerebral artery contain CGRP. Several possible developmental mechanisms underlying this enrichment were investigated. Some of this enrichment is accounted for by a prenatal selection of CGRP cells in the cerebrovascular projection. The remainder of the enrichment can be explained by a late (postnatal days 55-90) target-induced expression of CGRP in some trigeminal neurons innervating cerebral arteries. Most surprisingly, the massive postnatal regression in the trigeminal projection to the cerebral arteries (between postnatal days 5 and 55 , cell death and axon retraction delete $3 / 4$ of the neurons that innervate the middle cerebral artery neonatally) has no role in the CGRP enrichment in the cells remaining at maturity in this projection. These regressive events appear to affect equally the CGRP-positive and CGRP-negative populations. However, axon retraction is involved in the postnatal loss of CGRP enrichment seen in 1 small subpopulation of the trigeminal projection. Each trigeminal cell in this population sends axon collaterals to both the cerebral artery and the forehead skin neonatally, and then later most of these dually projecting neurons retract only their artery collaterals but do not die. A low percentage of CGRP-containing neurons does not appear to predate artery collateral retraction. Rather, this subpopulation of dually projecting neurons is enriched in CGRP neonatally, but loses the CGRP enrichment postnatally as some neurons are respecified to a non-CGRP phenotype after they retract their artery collaterals. Postnatal maintenance of a projection to a visceral target, the cerebral arteries, appears to preserve a neonatal CGRP enrichment and ensure a subsequent postnatal increase in CGRP expression in trigeminal neurons.

In the vertebrate peripheral nervous system distinct neurotransmitter profiles characterize mature sensory projections to different peripheral targets (Dockray and Sharkey, 1986; Gibbins

\footnotetext{
Received Dec. 11, 1989; revised March 12, 1990; accepted April 2, 1990.

This work was supported by the Ontario Heart and Stroke Foundation.

Correspondence should be addressed to Kevin Horgan, Neurobiology Research Group, Department of Anatomy, University of Toronto, Medical Sciences Building, Room 1105, Toronto, Ontario, Canada M5S $1 \mathrm{A8}$.

Copyright $\odot 1990$ Society for Neuroscience $0270-6474 / 90 / 072485-08 \$ 03.00 / 0$
}

et al., 1987; McMahon and Gibson, 1987; Molander et al., 1987). The trigeminal ganglion, which provides sensory innervation to cutaneous, muscular, and visceral targets in the head, contains a variety of neurotransmitters, including substance $P$ (Sub P), cholecystokinin (CCK), somatostatin (SOM), vasointestinal peptide (VIP), and calcitonin gene-related peptide (CGRP) (Jancso et al., 1981; Rosenfeld et al., 1983; O'Connor and van der Kooy, 1988). Trigeminal sensory projections to the periphery have been shown to have distinct neurotransmitter profiles with respect to the type of target innervated (Terenghi et al., 1986; Ositelu et al., 1987; O'Connor and van der Kooy, 1988). Multiple mechanisms may underlie the emergence of a mature, target-specific, transmitter profile in developing trigeminal projections. First, nerve-target matching may occur through precise initial projections of neurotransmitter-committed nerves to their appropriate targets. Second, neurotransmitter-unspecified afferents arriving at a peripheral target tissue may have their neurotransmitter phenotype directly specified by that target tissue. Third, the selective survival of neurotransmitter specific projecting nerves during the developmental cell death and axon retraction period may indirectly determine mature neurotransmitter profile. Results from several studies suggest direct target regulation of chemical phenotype (Marusich et al., 1986; McMahon and Moore, 1988; Philippe et al., 1988) and, more specifically, of neurotransmitter phenotype (McMahon and Gibson, 1987; Schotzinger and Landis, 1988; Horgan and van der Kooy, 1989; Stevens and Landis, 1990) in neurons.

The adult trigeminovascular projection is enriched in afferents containing the neurotransmitter CGRP both in comparison to the level of CGRP in the cutaneous trigeminal projection to the forehead skin and in comparison to the levels of other neuropeptides in the cerebral artery projection ( $\mathrm{O}^{\prime}$ Connor and van der Kooy, 1988). The trigeminal ganglion is an excellent system in which to study the development of putative target-directed neurotransmitter specificity, because the ganglion perikarya projecting to the cerebrovasculature and those projecting to the forehead skin are intermixed in the ophthalmic division of the ganglion (O'Connor and van der Kooy, 1986a). Thus, the cell body environments of the 2 types of perikarya seem similar, although their target projections (visceral versus cutaneous) and neurotransmitter profiles are very different. In addition, a subpopulation of these trigeminal cells projects to both artery and forehead neonatally, with almost all of these dually projecting cells retracting artery collaterals postnatally. This subpopulation of cells is an ideal system in which to study the effects of changing peripheral projections on neurotransmitter profile.

In the rat trigeminal ganglion afferents first contact their pe- 

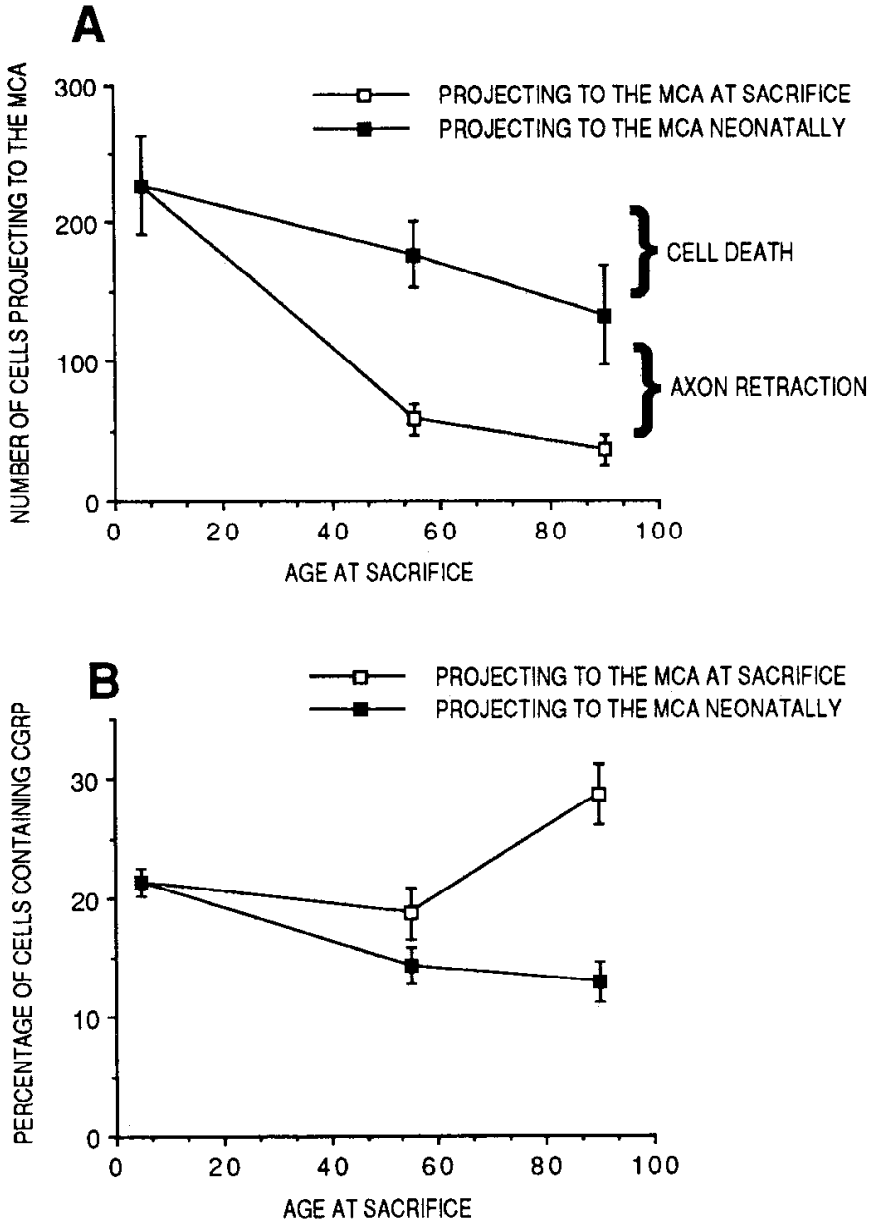

Figure 1. A, Regressive events among the trigeminal cells innervating the MCA. After a TB application to the MCA at $\mathrm{P} 3$ (cells projecting to the MCA neonatally), the number of retrogradely labeled TB cells decreases with age. By P90, approximately $40 \%$ of these trigeminal cells have died. Surviving cells in this population may either maintain an artery collateral or retract from the artery environment. TB applications to the MCA 2-4 d prior to killing (cells maintaining MCA projections postnatally) label the subpopulation of cells specifically maintaining an artery projection. The cells in this population reflect those remaining after both axon retraction and cell death. $B$, Percentage CGRP expression within the same populations of trigeminal cells described in $A$ that project to the MCA neonatally, and that specifically maintain MCA projections postnatally. Data represent means \pm SEM for each group. The number of rats in each group is: in the neonatally projecting population, $\mathrm{P} 5, n=8 ; \mathrm{P} 55, n=6$; $\mathrm{P} 90, n=7$; and in the population maintaining artery projections postnatally, P55, $n=7$; P90, $n=7$.

ripheral targets at embryonic day (E) 14-15 (Erzurumlu and Killackey, 1983), and CGRP expression in the peripheral processes of trigeminal artery projecting afferents first occurs several days later, around E18-E19 (Tsai et al., 1989). In the mouse trigeminal ganglion a period of embryonic cell death occurs when approximately $50 \%$ of the peak embryonic number of trigeminal neurons is lost by postnatal day (P) 4 (Davies and Lumsden, 1984). This embryonic cell death probably occurs in the rat trigeminal ganglion as well. A period of postnatal cell death has been observed in the rat trigeminal ganglion, where approximately $20 \%$ of cells die between P0 and P90 (maturity) (O'Connor and van der Kooy, 1986b). The amount of trigeminal ganglion cell death varies according to peripheral projection. The visceral afferent projection to the cerebral arteries undergoes an $85 \%$ loss in the number of projecting cells between P5 and P90, due to the combined effects of cell death and axon retraction. In contrast, over the same period the trigeminal cutaneous projection to the forehead skin undergoes little or no cell death or axon retraction (O'Connor and van der Kooy, 1986b). In neonatal rats, there is a subpopulation of trigeminal cells with axon collaterals to both the cerebral arteries and forehead skin. By P90 almost all of these forehead afferent neurons no longer project to the artery. This indicates that axon retraction and/or cell death facilitates the functional segregation of trigeminal projections, preventing cutaneous afferents from maintaining projections to visceral targets (O'Connor and van der Kony, 1986b). Together these studies suggest that cell death and axon retraction are general features of trigeminal sensory development and may play a special role with respect to organizing the size and composition of the trigeminal cerebrovascular projection.

The adult enrichment of CGRP in the visceral artery projection, together with the disproportionately low CGRP presence in the cutaneous forehead projection ( $\mathrm{O}^{\prime}$ Connor and van der Kooy, 1988), raises the question of how target-specific neurotransmitter profile is regulated in trigeminal sensory afferent projections. The fact that CGRP enrichment is present in the trigeminal artery projection after a substantial cell death period, in contrast to a lack of cell death and disproportionately low level of CGRP in the forehead projection, led us to hypothesizc that the CGRP enrichment in the mature artery projection may be due to selective cell death and/or axon retraction of nonCGRP trigeminal vascular afferents during the postnatal period. In vitro experiments have demonstrated that in both sympathetic (Edgar et al., 1981; Rohrer et al., 1983) and sensory (Barde et al., 1980) neuronal cultures distinct subpopulations differ in their survival factor requirements during development. Additional in vitro experiments have shown that neurotrophic factors released from corneal epithelium selectively support the survival and neurite outgrowth of only a subpopulation of neonatal trigeminal neurons from the ophthalmic division of the ganglion (the corneal epithelium is a peripheral target for neurons from the ophthalmic division of the trigeminal ganglion) (Chan and Haschke, 1981). In the present study we ask 2 questions: (1) Is the adult enrichment of CGRP in the trigeminal artery projection due to the selective survival of CGRP-containing affercnts at the artery? (2) Does axon retraction from the artery environment cause a loss of CGRP enrichment in surviving trigeminal neurons that retain axonal projections to the forehead?

\section{Materials and Methods}

The offspring of pregnant albino Wistar rats were used for all experiments. Rat pups were recorded as P0 on day of birth.

Maturation of the trigeminal projection to the cerebral arteries. P3 rat pups were anesthetized using cold temperatures. From a parietotemporal approach, the right middle cerebral artery (MCA) was isolated just ventral to the rhinal sulcus using a Zeiss operating microscope. Two parallel cuts were made through the dura and pia arachnoid on either side of the artery and a small piece of parafilm (approximately $1 \mathrm{~mm}$ $\times 4 \mathrm{~mm}$ ) was placed under the artery to limit tracer spread. A small amount of powdered True blue (TB) or Fast blue (FB) was applied directly to the artery and then covered by 1 or 2 pieces of Gelfoam. Animals were sutured and rewarmed until they recovered and allowed to survive to P5, P55, or P90. FB was used because in a previous study (O'Connor and van der Kooy, 1988) it appeared to yield brighter fluorescent labeling than TB in the cell bodies of the sympathetic superior cervical ganglion projection to the MCA (O'Connor and van der Kooy, 1988). However, in the present experiment TB and FB applications yielded similar numbers of retrogradely labeled trigeminal cells; there- 
fore, data collected with both tracers were combined. To differentiate cell death from axon retraction postnatally, siblings (of the P3 operated rat pups described above) did not receive a neonatal tracer application to the MCA. These siblings survived to P55 or P90, but at P52 or P86, respectively, these previously unoperated rats received a $T B$ application to the MCA exactly as described above (anesthetic used was sodium pentobarbitol, $65 \mathrm{mg} / \mathrm{kg}$ ) to specifically label the population of trigeminal cells maintaining an artery projection at the time of killing. In contrast, the neonatal tracer applications labeled surviving cells which projected to the artery at P3 and either maintain or retract their artery collateral by the time of sacrifice.

Maturation of the population of trigeminal cells with neonatal projections to the artery and forehead. Rat pups that received neonatal TB or FB applications to the MCA at P3 were allowed to survive until P5 or P90. One day prior to sacrifice, Diamidimo yellow (DY) was used to label the ipsilateral trigeminal projection to the forehead skin in these animals. To label the trigeminal projection to the forehead skin anesthetized animals had the frontal branch of their right ophthalmic nerve dissected free of surrounding tissue and cut just distal to the supraorbital foramen. A small piece of Gelfoam soaked in DY was placed on the proximal stump of the cut nerve and surrounded by dry Gelfoam. The animals were then sutured and allowed to recover. TB and FB fluorescence both appear as a characteristic blue color visible throughout the cytoplasm of labeled cells under a dark-field microscope illuminated at $360 \mathrm{~nm}$, whereas DY fluoresces light yellow under this same wavelength and is localized in the nucleus of labeled cells, making it easy to detect double-labeled cells.

Tissue processing and immunocytochemistry. At time of killing animals were deeply anesthetized with sodium pentobarbitol and decapitated. The right trigeminal ganglion of all rats was removed, washed, and then cultured in Dulbecco's modified Eagle's medium containing $10^{-4} \mathrm{M}$ colchicine for 2-3 $\mathrm{hr}$ (neonatal ganglia) or 5-6 hr (adult ganglia). Colchicine pretreatment improves visualization of neurotransmitter immunohistofluorescence without increasing the overall number of transmitter positive cells identified in the trigeminal ganglion ( $\mathrm{O}^{\prime} \mathrm{C}$ Connor and van der Kooy, 1988) and was used to reduce variability in the scoring of CGRP-positive cells. Ganglia were then immersed in $4 \%$ paraformaldehyde in $0.1 \mathrm{~m}$ phosphate-buffered saline (PBS) for $24 \mathrm{hr}$ at $4^{\circ} \mathrm{C}$ and then transferred to $20 \%$ sucrose for another $24 \mathrm{hr}$ at $4^{\circ} \mathrm{C}$. Sections of $24 \mu \mathrm{m}$ or $32 \mu \mathrm{m}$ were cut on the cryostat. Trigeminal ganglion sections were incubated at $4^{\circ} \mathrm{C}$ for $24 \mathrm{hr}$ with primary antiserum to CGRP (Peninsula, against Tyr CGRP) diluted to 1:300 in 0.1 M PBS containing $1 \%$ normal goat serum (NGS) or normal horse serum (NHS) and $0.3 \%$ Triton X100. After primary incubation the ganglia were rinsed 2 or 3 times (rinsing solution: 0.1 m PBS containing 1\% NGS or NHS and $0.3 \%$ Triton $\mathrm{X} 100$ ) and then incubated at room temperalure in goat or sheep anti-rabbit IgG conjugated with fluorescein isothiocyanate (Cappel) diluted 1:50. After a final rinse with $0.1 \mathrm{M}$ PBS, sections were mounted on gel-coated slides. Sections were counted with an epifluorescent Leitz microscope using a $360 \mathrm{~nm}$ filter for TB, FB, and DY labeling and a $470 \mathrm{~nm}$ filter for CGRP staining. All labeled cells in the ipsilateral ganglia were counted and these counts were corrected using the method of Abercrombie (1946). Absorption controls demonstrated the specificity of the CGRP antiserum by the abrogation of immunostaining in ganglion sections incubated in primary antiserum to CGRP together with the CGRP peptide itself (Peninsula).

\section{Results}

\section{Postnatal cell death and axon retraction in the trigeminal} artery projection

The present results replicate previous findings (O'Connor and van der Kooy, 1986b, 1989) in showing a significant $(t=2.01$, $n=15, p<0.05$ ) cell loss between P5 and P90 in the population of trigeminal cells with neonatal cerebral artery projections (retrogradely labeled with a tracer application to the MCA at P3) (Fig. 1A). The smaller number of trigeminal MCA projecting cells sampled in the present report as compared to previously published results is probably due to a more distal tracer application site on the MCA (sensory innervation density along the MCA decreases distally (Tsai et al., 1989)). Approximately 40\% of cells present at P5 have died by P90. This cell loss is likely due to naturally occurring cell death. Previous findings ( ${ }^{\prime}$ Con- nor and van der Kooy, 1986b) show that there is virtually no loss over the same period in the population of trigeminal ganglion cells retrogradely labeled with a neonatal tracer application to the forehead. These results negate fluorescent tracer cytotoxicity in trigeminal cells and also indicate that TB does not leak appreciably during long-term survival experiments. 'I he number of trigeminal cells specifically maintaining an artery projection postnatally (labeled by a TB application to the MCA just prior to killing) decreases significantly from P5 to P90 ( $t=5.34$, $n=15, p<0.05$ ) (Fig. 1A). There are fewer trigeminal cells specifically maintaining artery projections at both P55 and P90 in comparison to the number of cells that had neonatal artery projections and remain alive at these later times. This indicates that a subpopulation of the surviving neonatally artery projecting neurons retracts axon collaterals from the artery environment postnatally. Thus axon retraction as well as cell death accounts for the postnatal regression in the artery projection. Most of this axonal retraction occurs between P5 and P55. In summary, $40 \%$ of the cells projecting to the MCA at P5 undergo cell death by $\mathrm{P} 90$, a further $40 \%$ retract axon collaterals from the MCA by $\mathrm{P} 90$.

In these experiments we have assumed that tracer application at different ages results in a uniform sampling of nerves projecting to the MCA. Several points support this assumption. First, the branching pattern of the MCA is characteristic and tracer applications were made to the same place on the artery at different ages. Second, tracer spread was minimized using a combination of parafilm and Gelfoam to localize TB crystals to the artery. Third, 2 points suggest that TB does not diffuse into the MCA to be picked up by trigeminal neurons at alternate targets. It has been demonstrated that TB does not easily gain access to the arterial lumen after application to the outside of the artery (O'Connor and van der Kooy, 1986b). Furthermore, cauterization of the MCA proximal to the site of tracer application abolishes TB labeling in the trigeminal ganglion ( $\mathrm{O}^{\prime} \mathrm{Con}-$ nor and van der Kooy, 1986a), indicating that only those nerves coursing distally along the artery at the application site are labeled.

\section{Incidence of CGRP in the maturing trigeminal artery projection}

At P5, 21\% of artery projecting cells contain CGRP (Fig. 1B). This substantial presence of CGRP in the neonate increases significantly by P90 ( $t=2.87, n=15, p<0.05)$, when $29 \%$ of trigeminal cells specifically maintaining an artery projection at P90 contain CGRP. However, this postnatal increase occurs entirely between P55 and P90 $(t=3.25, n=14, p<0.05)$, at a time when almost all of the cell death and axon retraction in this projection has already occurred (Fig. $1 \mathrm{~A}$ ). Prior to P55 there is no significant postnatal change in the percentage of CGRP cells maintaining a projection to the cerebral arteries $(t=1.24$, $n=15, p>0.05$ ), yet this is the period when most of the postnatal cell death and axon retraction occurs in this same population. These results strongly suggest that the CGRP enrichment in the mature trigeminal artery projection is not due to differential cell death and axon retraction; rather, it is due to the substantial percentage of trigeminal cells containing CGRP that already project to the MCA in the neonate, as well as to the increase in the percentage of MCA projecting cells containing CGRP occurring in the period after the majority of the postnatal cell death and axon retraction has finished. 

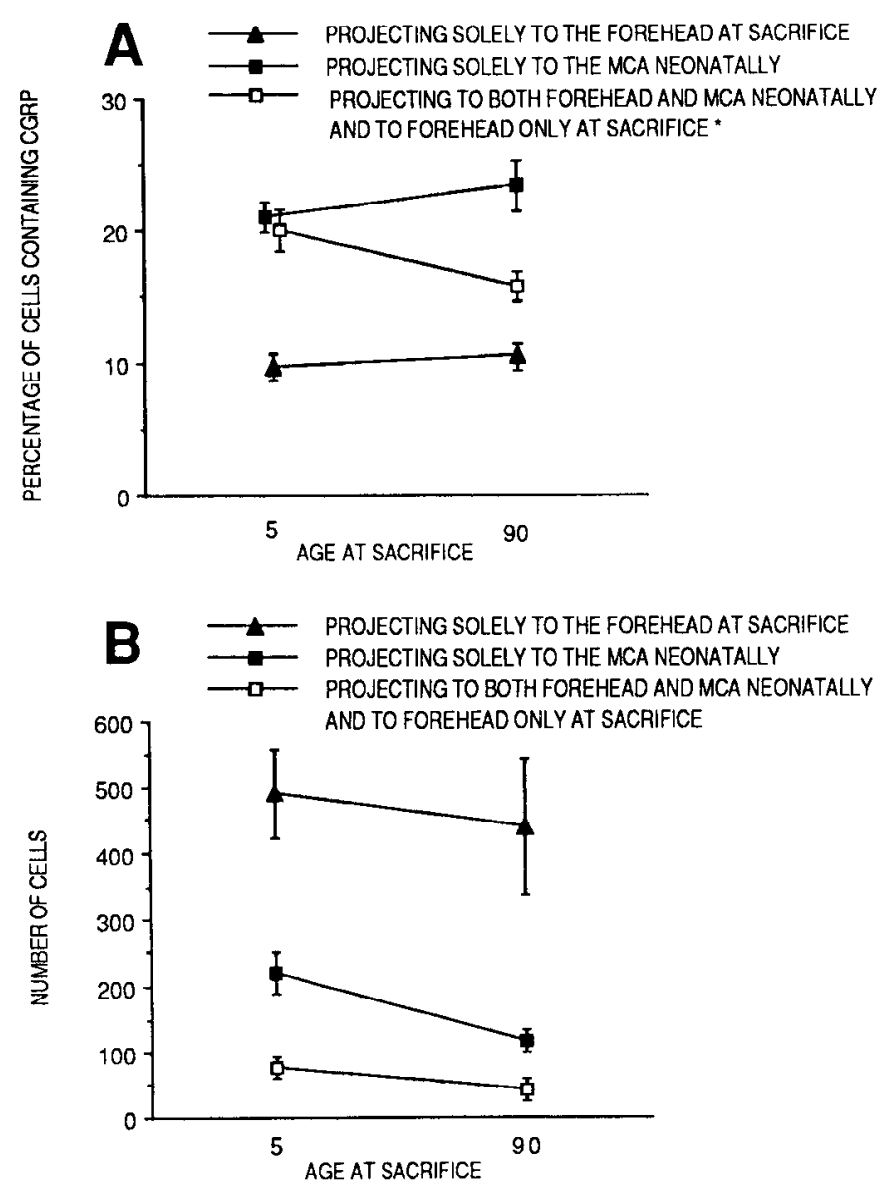

Figure 2. A, Postnatal incidence of CGRP in 3 populations of trigeminal cells: cells projecting solely to the forehead skin at killing, cells projecting solely to the MCA neonatally, and cells with neonatal projections to both forehead and MCA which retract their MCA collateral postnatally. Note that the latter 2 groups are together equivalent to the population of cells projecting to the MCA neonatally shown in Figure 1. $B$, Postnatal cell numbers in the same artery projecting, forehead projecting, and dually projecting trigeminal populations described in $A$. Data represent means \pm SEM for each group of rats. The number of rats per group $=11$, at both $\mathrm{P} 5$ and P90. ${ }^{*}$ On average, less than 1 trigeminal cell per animal projects to both the MCA and the forehead skin at maturity (O'Connor and van der Kooy, 1986b). Therefore, we assume that the population of neonatally artery projecting trigeminal cells that projects to the forehead at maturity has retracted its artery projections postnatally. Since there is no significant cell death or axon retraction in the trigeminal forehead skin projection postnatally, we assume that forehead projecting cells labeled in the adult are also present neonatally.

\section{Incidence of CGRP in trigeminal cells retracting their artery projection postnatally}

There is a significant decrease $(t=4.33, n=15, p<0.05)$ between P5 and P90 in the percentage of CGRP-containing neurons in the population of trigeminal cells with a neonatal projection to the cerebral artery; $21 \%$ of these cells are CGRP positive at P5 but only 13\% contain CGRP by P90 (Fig. $1 B$ ). The decrease in CGRP in this population occurs predominantly betwcen P5 and P55, when the percentage of CGRP-positive cells drops from $21 \%$ to $14 \%(t=3.97, n=14, p<0.05)$. This population of cells is a composite of cells either retracting or maintaining artery collaterals postnatally but not dying. It appears that cells retracting their axons may be responsible for the decrease in incidence of CGRP for 2 reasons. First, the period between P5 and P55 when CGRP enrichment is lost in this population coincides with the period over which neurons are retracting their MCA collaterals (Fig. 1A). Second, over this same period the subpopulation of cells specifically maintaining an artery projection undergoes no change in the percentage of CGRP-positive cells. Therefore, axon collateral retraction from the artery environment correlates with loss of CGRP enrichment. There are 2 possible ways in which axon retraction and CGRP expression may be related. First, collateral retraction from the cerebral artery environment may cause loss of CGRP enrichment, either through differential cell death of CGRP-containing cells in the retracting population or through respecification of CGRP-containing cells to the non-CGRP phenotype after artery collateral retraction. Alternatively, the population fated to retract its MCA collaterals may already be low in CGRP while projecting neonatally to the artery. If the sccond possibility were correct, then artery collateral retraction by a population of cells low in CGRP should be reflected in a concurrent rise in CGRP level in the complementary population of cells remaining at the artery. However, no increase is seen in CGRP-positive neurons between P5 and P55 in the population specifically retaining a MCA projection. Therefore, we suggest that loss of an artery collateral causes a decrease in CGRP expression, and that this population fated to withdraw from the artery environment is not already low in CGRP prior to retraction. It is not possible to directly test these 2 distinct possibilities in the present paradigm, since information about postnatally retracting cells is only obtained indirectly.

\section{CGRP in trigeminal cells with neonatal projections to artery and forehead skin}

The trigeminal ganglion contains a subpopulation of neurons that project neonatally to both the cerebral arteries and the forehead skin. Almost all these cells retract their artery collateral postnatally (O'Connor and van der Kooy, 1986b) and in the adult project solely to the forehead skin, a target whose overall projection from the trigeminal ganglion is low in CGRP-positive cells (O'Connor and van der Kooy, 1988). Monitoring CGRP over postnatal development in this specific population should reveal whether loss of CGRP enrichment occurs as artery collaterals retract.

At $\mathrm{P} 5$, the population of trigeminal cells with dual projections to the artery and forehead skin (double labeled with 2 different retrograde tracers applied to the MCA and to the forehead) contains 20\% CGRP-positive cells (Fig. 2A), a level that is not significantly different from that seen in the population projecting only to the MCA at this time $(t=0.13, n=22, p>0.05)$. Moreover, these dually projecting cells have a CGRP level that is significantly higher than the 10\% CGRP level in the population of trigeminal cells projecting only to the forehead at this age $(t=6.22, n=22, p<0.05)$. At maturity (P90), the level of CGRP in this double-labeled population, now projecting solely to the forehead skin, has significantly decreased from $20 \%$ to $16 \%$ CGRP-positive cells $(t=2.5, n=22, p<0.05)$. This adult level is also significantly lower than the $23 \%$ CGRP cells seen in the MCA only projecting population at this age $(t=3.64, n$ $=22, p<0.05$ ). These results suggest that artery collateral retraction causes loss of CGRP enrichment postnatally in the retracting population. However, axon retraction from the middle cerebral artery does not appear to cause a complete loss of CGRP enrichment, since the population that retracts artery col- 
laterals postnatally but retains a forehead skin projection at maturity is still significantly $(t=4.03, n=22, p<0.05)$ higher in CGRP-containing cells (16\% CGRP positive) than the population projecting only to the forehead skin at maturity $(10 \%$ CGRP positive). Perhaps some of the dually projecting cells have become irrevocably committed to the CGRP phenotype by the time they retract from the artery. The loss in CGRP enrichment after axon retraction from the MCA could be explained either by preferential cell death of CGRP-containing cells no longer projecting to the artery or by respecification of CGRP-positive cells to the non-CGRP phenotype after loss of artery collaterals.

In the population of trigeminal cells projecting solely to the MCA neonatally in Figure 2, there is no postnatal loss of CGRP enrichment. This neonatal MCA only projection shown in Figure 2 is a subpopulation of the neonatal MCA projection shown in Figure 1. Absent are cells with dual neonatal projections to both forehead skin and artery, which retract their artery collaterals postnatally. The absence of these postnatal artery retracting cells from the group in Figure 2 projecting solely to the MCA neonatally presumably explains why, in contrast to the results for the neonatal MCA projection in Figure 1, there is no postnatal loss of CGRP enrichment in this Figure 2 group.

\section{Postnatal cell death in artery and forehead innervating cells}

A large number of cells project to both the cerebral artery and forehead skin in the neonate. However, it has been shown previously that only a rare cell projects to both the MCA and forehead skin in the adult (O'Connor and van der Kooy, 1986a). Axon retraction is a feature of the trigeminal cerebral artery projection (Fig. $1 A$ ), yet there is no significant axon retraction or cell death in the population of trigeminal neurons projecting only to the forehead skin between P5 and P90 ( $t=0.37, n=$ $22, p>0.05$ ) (Fig. 2B). Taken together, these results predict that most cells with neonatal projections to both cerebral artery and forehead skin postnatally retract their MCA projection, but maintain their forehead skin projection and do not die. This is confirmed by the fact that the number of cells that project to both the artery and the forehead skin at P5 is not significantly higher than the number of cells with neonatal artery projections that retain a projection to the forehead skin at $\mathrm{P} 90(t=1.51, n$ $=22, p>0.05)$. The observation that this population suffers a loss of CGRP-containing cells after artery collateral retraction, and yet undergoes no significant cell death, suggests that CGRPcontaining cells are respecified to the non-CGRP phenotype after axonal retraction from the artery environment.

\section{Discussion}

CGRP is present in 29\% of ganglion perikarya projecting to the adult MCA. Figure 3 schematizes 3 possible developmental mechanisms (A, B, and $C$ ) that may underlie this enrichment. Most surprisingly, the results revealed that this CGRP enrichment is not due to the selective survival of CGRP-containing afferents in the face of the massive postnatal cell death and axon retraction (mechanism B) that characterizes this projection (O'Connor and van der Kooy, 1986b, 1989). Instead, the enrichment is a result of the $21 \%$ CGRP-containing cells present in this projection neonatally (mechanism $\mathrm{A}$ ), together with a late postnatal increase in percentage of CGRP-containing cells up to the adult level of $29 \%$ (mechanism C) that takes place entirely between P55 and P90 (when most of the postnatal cell death and axon retraction has already occurred). A contribution of selective cell death and differential axon retraction to the postnatal CGRP enrichment in the artery projection cannot be conclusively ruled out. However, 2 results suggest that target specification of neurotransmitter phenotype and target regulation of afferent neuron number may occur independently in this projection. First, the percentage of CGRP-containing cells in the artery projection remains constant between P5 and P55, a period during which most of the postnatal cell death and axon retraction occurs in this projection. Second, in the population of trigeminal cells with neonatal projections to both cerebral artery and forehead skin that is fated to retract its artery collaterals by maturity, CGRP level prior to retraction from the artery environment is the same as that seen in the overall artery projection. This suggests that differential axon retraction of a subpopulation of artery projecting cells low in CGRP is not a contributing factor to the increase in CGRP enrichment seen in the artery projection postnatally.

Retraction of axon collaterals from the artery environment causes a loss of CGRP enrichment postnatally (mechanism D). This is not formally a mechanism that enriches CGRP in the adult artery projection, but instead is a mechanism that decreases CGRP expression in neurons that retract axons from the artery environment postnatally. The population of trigeminal afferents with nconatal projections to both ccrcbral artery and forehead skin contains the same percentage of CGRP-positive cells as the overall artery projection at P5 (20\%). CGRP enrichment is lost only after these cells retract from the artery environment, with CGRP-positive ganglion cells dropping to $16 \%$ of the retracting population by maturity. Respecification of some CGRP-containing cells to the non-CGRP phenotype after retraction from the artery environment appears to be the mechanism that produces loss of CGRP enrichment in this population, since there is no significant loss of cells in this population postnatally. It is interesting to note that after axon retraction from the artery, this population which projects solely to the forehead skin at P90 still has a significantly higher level of CGRP (16\%) than that seen in the overall forehead skin projection $(10 \%)$ at $\mathrm{P} 90$. Perhaps this is a result of relatively late maintenance of some artery collaterals through a critical period of neurotransmitter plasticity in this afferent population. In this scenario, only early postnatal retraction from the artery environment results in respecification of CGRP-containing cells to the non-CGRP phenotype. Later-retracting CGRP-positive afferents would maintain their CGRP phenotype, resulting in a mixed population of trigeminal cells which either were respecified to the non-CGRP phenotype after artery collateral retraction or maintained neonatal CGRP levels after retraction. The present results indicate that the bulk of both cell death and axon retraction in the artery projection occurs prior to P55, yet previous experiments have also revealed that an early period of axon retraction occurs together with cell death prior to P25, while a smaller and later period of axon retraction without cell death occurs between P25 and P55 (O'Connor and van der Kooy, 1989). Perhaps the subpopulation of neurons retracting from the artery environment later in development (P25-P55) are irreversibly committed to their CGRP neurotransmitter phenotype prior to retraction. It has been suggested in other studies that neurotransmitter plasticity in peripheral nerves decreases developmentally (Hill and Hendry, 1977; Ross et al., 1977).

A recent report on the postnatal development of CGRP-containing terminal plexes on the cerebral arteries shows that there 


\section{EARLY CGRP PRESENCE}
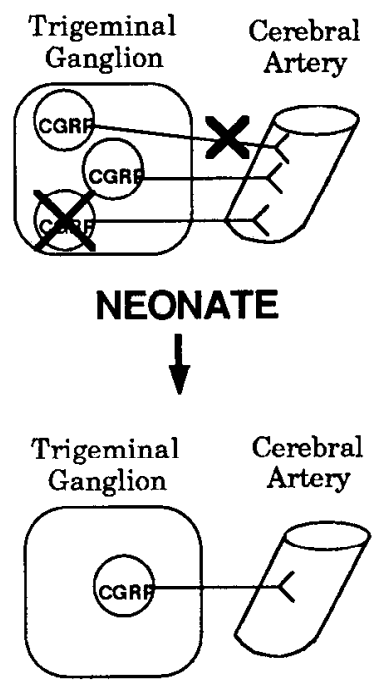

ADULT

\section{PASSIVE CGRP ENRICHMENT DUE TO REGRESSION OF OTHER PROJECTIONS}
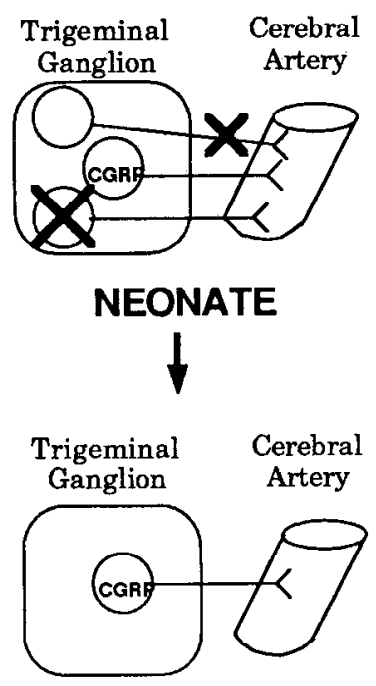

ADULT

\section{ACTIVE CGRP ENRICHMENT AFTER REGRESSION OF OTHER PROJECTIONS}
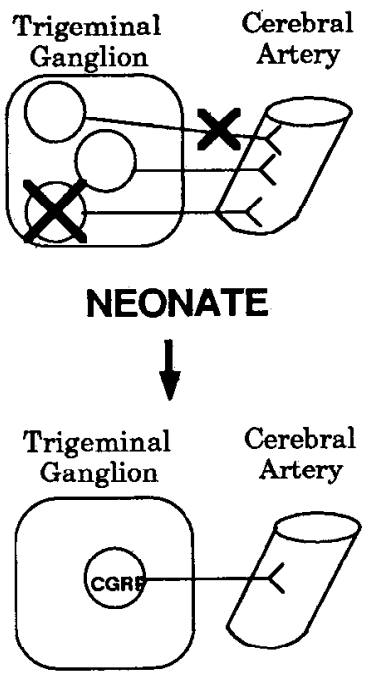

ADULT
ACTIVE CGRP

SUPPRESSION AFTER

REGRESSION OF ARTERY COLLATERALS
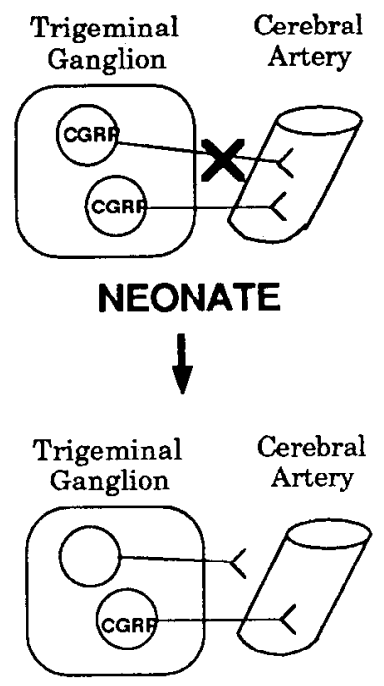

ADULT

\section{A}
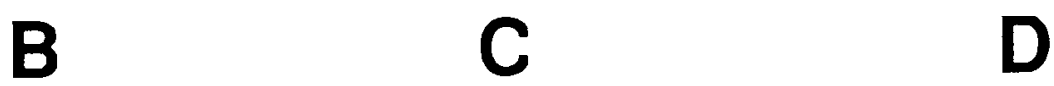

Figure 3. Four possible developmental mechanisms underlying the enrichment of CGRP in the trigeminal artery projection $(A, B, C)$ and the loss of CGRP enrichment after the retraction of artery collaterals $(D)$. The present study has provided evidence for mechanisms $A$, $C$, and $D$, and against mechanism $B$. See text for details.

is a substantial increase in the density of CGRP fibers on the cerebral arteries up until P30-31, when the adult pattern and fiber density of CGRP-positive fibers on the cerebral arteries is achieved (Tsai et al., 1989). The trigeminal ganglion is the major source of these CGRP fibers (McCulloch et al., 1986). Thus the postnatal regression (cell death and axon retraction) of the trigeminal sensory projection to the cerebral arteries (which occurs largely before P25 (O'Connor and van der Kooy, 1989)) happens concurrently with the developmental increase of CGRP terminal fiber density on the cerebral arteries. This raises the question of whether these 2 processes are independent. Perhaps survival and maintenance of a single trigeminal neuron's sensory projection requires extensive terminal fiber branching on the cerebral arteries during the developmental regression period. The fact that terminal CGRP fiber density increases markedly on the cerebral arteries during the time over which the majority of the early CGRP projections are lost seems to indicate that cells with surviving projections must dramatically increase their terminal arborizations, or at least CGRP expression in these arborizations, over this time. Studies on synaptogenesis at the neuromuscular junction (Nystrom, 1968; Brown et al., 1976; Riley, 1976) and in peripheral ganglia (Lichtman, 1977; Johnson and Purves, 1981) reveal similar results. In both these systems there is an overall increase in synaptic density during the neonatal period of elimination of polyneural innervation. Since this increase occurs when there is no increase in the total number of motor units or preganglionic inputs and since it occurs in spite of the fact that axonal projections are becoming more restricted, these studies suggest that neurons retaining original projections must increase their terminal arborizations markedly at this time.

The neonatal presence of CGRP in $21 \%$ of trigeminal neurons projecting to the cerebrovasculature and in $10 \%$ of trigeminal neurons projecting to the forehead skin indicates that neurotransmitter differentiation occurs prenatally in the trigeminal ganglion and that there is already some target specificity of transmitter phenotype in the afferent populations of the neonate. In the rat trigeminal system it has been shown that peripheral fibers reach their targets as early as E14-15 (Erzurumlu and Killackey, 1983) and CGRP expression in the terminal arborizations of trigeminal fibers innervating the cerebral arteries is first observed at E18-19 (Tsai et al., 1989). In the mousc, a period of prenatal cell death has been observed in the trigeminal ganglion, where approximately $50 \%$ of trigeminal cells die between E13 and the early postnatal period (Davies and Lumsden, 1984). Therefore, it is possible that selective prenatal cell death of non-CGRP-positive neurons may account for the $21 \%$ enrichment of CGRP-positive neurons seen in the trigeminal cerebrovascular projection at P5. Alternatively, precise prenatal projections of CGRP-committed trigeminal cells to correct peripheral targets may underlie the target-specific differences in neurotransmitter phenotype. Two findings from the present study argue against the initial directed outgrowth of neurotransmitter committed afferents playing a decisive role in shaping mature transmitter profile in trigeminal projections. First, the large number of trigeminal cells with neonatal forehead and artery 
projections that retract their artery axon collateral suggests that the maturation of projections in this system involves substantial postnatal rearrangement of the initial pattern of fiber terminations. Second, the respecification of some CGRP-containing neurons to the non-CGRP phenotype after withdrawal of an axon collateral from the artery environment indicates that early phenotypic specification with respect to neurotransmitters can be overridden.

A major finding of this report is that differential postnatal cell death and axon retraction do not directly modify the neurotransmitter profile of trigeminal projections to the cerebrovasculature. Competition among an excess of projecting neurons for target synaptic space or target released trophic molecules has been proposed to regulate these naturally occurring regressive events to produce a quantitative matching between nerve projection and target (Oppenheim, 1981; Cowan et al., 1984). Nerve growth factor (NGF) is the trophic factor best characterized to date, supporting the survival of sensory and sympathetic neurons during development. NGF also appears to regulate neurotransmitter synthesis in sensory and sympathetic neurons (Chun and Patterson, 1977; Mudge, 1981); however, evidence suggest that NGF controls only the quantity of neurotransmitter produced and does not specify neurotransmitter phenotype (Chun and Patterson, 1977; Patterson, 1978). Evidence for a strictly permissive role for NGF in transmitter differentiation comes from studies on sympathetic neurons where NGF promotes the synthesis of both acetylcholine and noradrenaline, while separate factors specify the switch in transmitter phenotype between acetylcholine and noradrenaline (Chun and Patterson, 1977). These results, along with those in the present study, suggest independent trophic and transmitter specifying influences on sensory neuron development. Nevertheless, it is possible that the postnatal cell death and axon retraction in the trigeminal cerebrovascular projection are indirectly responsible for the late postnatal increase in CGRP seen in this projection. Early postnatal loss of trigeminal artery projecting cells might allow remaining projecting cells greater access to putative transmitter-specifying molecules present at the artery, bringing about the late postnatal increase in CGRP enrichment seen in this projection.

Target regulation of chemical phenotype during ontogeny has been suggested to occur in both the autonomic and sensory nervous systems, where changes in the expression of biochemical markers (Marusich et al., 1986; Philippe et al., 1988), and more specifically neurotransmitter phenotype (Landis, 1983; Landis et al., 1988), in afferent populations correlate with onset of target innervation or exposure to target-derived factors. For example, sympathetic neurons that innervate sweat glands in rat footpads are noradrenergic prior to reaching their target, but undergo a transition to the cholinergic phenotype after contacting the sweat gland (Landis, 1983). In vitro experiments on isolated single sympathetic neurons have demonstrated that these nerves can switch between noradrenergic and cholinergic depending on culture conditions (Potter et al., 1986). Together these experiments strongly suggest that the target tissue respecifies noradrenergic nerves to the cholinergic phenotype. Definitive in vivo experiments addressing this question have shown that transplant of the cholinergic target footpad into the path of previously noradrenergic sympathetic afferents to the skin respecifies these nerves to the cholinergic phenotype (Schotzinger and Landis, 1988). Corresponding experiments altering the peripheral targets, cutaneous versus visceral or muscular, of sensory nerves have also shown some respecification of transmitter phenotype to that appropriate for the new target (McMahon and Gibson, 1987; Horgan and van der Kooy, 1989).

CGRP is enriched in the mature trigeminal projection to the cerebral arteries. This enrichment is due to a relatively high percentage of CGRP present in this specific projection neonatally, together with a late postnatal increase in CGRP expression. This late postnatal increase in the number of CGRP-positive cells in the trigeminal projection to the cerebral vasculature may be an active target-regulated process. Differential cell death and axon retraction do not appear to be directly responsible for the postnatal enrichment of CGRP in artery projecting cells, since the regression due to these processes is complete prior to the observed increase in CGRP-positive cells. Retraction of an axon collateral from the cerebral artery environment causes a loss of CGRP enrichment in trigeminal cells maintaining an axon to the cutaneous forehead target. This loss of enrichment appears to be due to the respecification of retracting CGRP-positive cells to the non-CGRP phenotype, since there is no significant cell death within the retracting population postnatally. The present results suggest that postnatal maintenance of a projection to a visceral target, the cerebral arteries, ensures CGRP enrichment in trigeminal neurons.

\section{References}

Abercrombie M (1946) Estimation of nuclear population from microtome sections. Anat Rec 94:239-247.

Barde, YA, Edgar D, Thoenen H (1980) Sensory neurons in culture: changing requirements for survival factors during embryonic development. Proc Natl Acad Sci USA 77:1199-1203.

Brown MC, Jansen JKS, van Essen D (1976) Polyneural innervation of skeletal muscle in new-born rats and its elimination during maturation. J Physiol 261:387-442.

Chan KY, Haschke RH (1981) Action of a trophic factor(s) from rabbit corneal epithelial culture on dissociated trigeminal neurons. J Neurosci 1:1155-1162.

Chun LLY, Patterson PH (1977) Role of nerve growth factor in the development of rat sympathetic neurons in vitro. J Cell Biol 75:712718.

Cowan WM, Fawcett FW, O'Leary DDM, Stanfield BB (1984) Regressive events in neurogenesis. Science 225:1258-1265.

Davies A, Lumsden A (1984) Relation of target encounter and neuronal death to nerve growth factor responsiveness in the devcloping mouse trigeminal ganglion. J Comp Neurol 223:124-137.

Dockray GJ, Sharkey KA (1986) Neurochemistry of visceral afferent neurones. Prog Brain Res 67:133-148.

Edgar D, Barde YA, Thoenen H (1981) Subpopulations of cultured chick sympathetic neurons differ in their requirements for survival factors. Nature 289:294-295.

Erzurumlu R, Killackey HP (1983) Development of order in the rat trigeminal system. J Comp Neurol 213:365-380.

Gibbins IL, Furness JB, Costa M (1987) Pathway-specific patterns of the coexistence of substance $\mathbf{P}$, calcitonin gene-related peptide, cholecystochinin and dynorphin in neurons of the dorsal root ganglion of the guinea pig. Cell Tissue Res 248:417-437.

Hill CE, Hendry HA (1977) Development of neurons synthesizing noradrenalin and acetylcholine in the superior cervical ganglion of the rat in vivo and in vitro. Neuroscience 2:741-749.

Horgan K, van der Kooy D (1989) The shithead experiments: visceral, but not cutaneous, targets dictate calcitonin gene-related peptide (CGRP) enrichment in trigeminal ganglion afferents. Soc Neurosci Abstr 15:1327.

Jancso G, Hokfelt T, Lundberg JM, Kiraly E, Halasz N, Nilsson G, Terenius L, Rehfeld J, Steinbusch H, Verhofstad A, Elde R, Said S, Brown M (1981) Immunohistochemical studies on the effect of capsaicin on spinal and medullary peptide and monoamine neurons using 
antisera to substance $P$, gastrin/CCK, somatostatin, VIP, enkephalin, neurotensin and 5-hydroxytryptamine. J Neurocytol 10:963-980.

Johnson DA, Purves D (1981) Postnatal reduction of neural unit size in the rabbit ciliary ganglion. J Physiol 318:143-159.

Landis SC (1983) Development of cholinergic sympathetic neurons: evidence of transmitter plasticity in vivo. Fed Proc 42:1633-1638.

Landis SC, Siegel RE, Schwab M (1988) Evidence for neurotransmitter plasticity in vivo. II. Immunocytochemical studies of rat sweat gland innervation during development. Dev Biol 126:129-140.

Lichtman JW (1977) The reorganization of synaptic connexions in the rat submandibular ganglion during postnatal development. J Physiol 273:155-177.

Marusich MF, Pourmehr K, Weston JA (1986) The development of an identified subpopulation of avian sensory neurons is regulated by interaction with the periphery. Dev Biol 118:505-510.

McCulloch J, Uddman R, Kingman TA, Edvinsson L (1986) Calcitonin gene-related peptide: functional role in cerebrovascular regulation. Proc Natl Acad Sci USA 83:5731-5735.

McMahon SB, Gibson S (1987) Peptide expression is altered when afferent nerves reinnervate inappropriate tissue. Neurosci Lett 74:915 .

McMahon SB, Moore CEG (1988) Plasticity of primary afferent acid phosphatase expression following rerouting of afferents from muscle to skin in the adult rat. J Comp Neurol 274:1-8.

Molander C, Ygge J, Dalsgaard CJ (1987) Substance P-, somatostatin-, and calcitonin gene-related peptide-like immunoreactivity and fluoride resistant acid phosphatase-activity in relation to regrogradely labeled cutaneous, muscular and visceral primary sensory neurons in the rat. Neurosci Lett $74: 37-42$.

Mudge AW (1981) Effect of chemical environment on levels of substance $P$ and somatostatin in cultured sensory neurones. Nature 292: 764-767.

Nystrom B (1968) Postnatal development of motor nerve terminals in "slow-red" and "fast-white" cat muscles. Acta Neurol Scand 44: 363-383.

O'Connor TP, van der Kooy D (1986a) Pattern of intracranial and extracranial projections of trigeminal ganglion cells. J Neurosci 6: 2200-2207.

O'Connor TP, van der Kooy D (1986b) Cell death organizes the postnatal development of the trigeminal innervation of the cerebral vasculature. Dev Brain Res 27:223-233.

O'Connor TP, van der Kooy D (1988) Enrichment of a vasoactive neuropeptide (calcitonin gene-related peptide) in the trigeminal sensory projection to the intracranial arteries. J Neurosci 8:2468-2476.

O'Connor TP, van der Kooy D (1989) Cooperation and competition during development: neonatal lesioning of the superior cervical ganglion induces cell death of trigeminal neurons innervating the cerebral blood vessels but prevents the loss of axon colaterals from the neurons that survive. J Neurosci 9:1490-1501.

Oppenheim RW (1981) Neuronal cell death and some related regressive phenomena during neurogenesis: a selective historical review and progress report. In: Studies of developmental neurobiology: essays in honour of Victor Hamburger (Cowan WM, ed), pp 74-133. New York: Oxford UP.

Ositelu DO, Morris R, Vaillant C (1987) Innervation of facial skin but not masticatory muscles or the tongue by trigeminal primary afferents containing somatostatin in the rat. Neurosci Lett 78:271276.

Patterson PH (1978) Environmental determination of autonomic neurotransmitter functions. Annu Rev Neurosci 1:1-17.

Philippe E, Garosi M, Droz B (1988) Influence of peripheral and central targets on subpopulations of sensory neurons expressing calbindin immunoreactivity in the dorsal root ganglion of the chick embryo. Neuroscience 26:225-232.

Potter DD, Landis SC, Matsumoto SG, Furshpan EJ (1986) Synaptic functions in rat sympathetic neurons in microcultures. II. Adrenergic/ cholinergic dual status and plasticity. J Neurosci 6:1080-1098.

Riley DA (1976) Multiple axon branches innervating single endplates of kitten soleus myofibers. Brain Res 110:158-161.

Rohrer H, Thoenen H, Edgar D (1983) Presence of nerve growth factor and catecholamine uptake in subpopulations of chick sympathetic neurons: correlation with survival factor requirements in culture. Dev Biol 99:34-40.

Rosenfeld MG, Mermod JJ, Amara SG, Swanson LW, Sawchenko PE, Rivier J, Vale WW, Evans RM (1983) Production of a novel neuropeptide encoded by the calcitonin gene via tissue specific RNA processing. Nature 267:536-539.

Ross D, Johnson M, Bunge R (1977) Development of cholinergic characteristics in adrenergic neurons in age dependent. Nature 267: 536-539.

Schotzinger RJ, Landis SC (1988) Cholinergic phenotype developed by noradrenergic sympathetic neurons after innervation of a novel cholinergic target in vivo. Nature 335:637-639.

Stevens LM, Landis SC (1990) Target influences on transmitter choice by sympathetic neurons developing in the anterior chamber of the eye. Dev Biol 137:109-124.

Terenghi G, Polak JM, Rodrigo J, Mulderry PK, Bloom SR (1986) Calcitonin gene-related peptide-immunoreactivity in the tongue, epiglottis and pharynx of the rat: occurrence, distribution and origin. Brain Res 365:1-14.

Tsai SH, Tew JM, Shipley MT (1989) Cerebral arterial innervation. II. Development of calcitonin gene-related peptide and norepinephrine in the rat. J Comp Neurol 279:1-12. 